\title{
Disk Galaxies at High Redshift?
}

\author{
Max Pettini ${ }^{1}$ \\ ${ }^{1}$ Institute of Astronomy, Madingley Road, Cambridge CB3 0HA, UK
}

\begin{abstract}
The successful implementation of integral field near-infrared spectrographs fed by adaptive optics is providing unprecedented views of gas motions within galaxies at redshifts $z=2-3$, when the universe was forming stars at its peak rate. A complex picture of galaxy kinematics is emerging, with inflows, rotation within sometimes extended and nearly always thick disks, mergers, and galaxy-wide outflows all contributing to the variety of patterns seen. On the computational side, simulations of galaxy formation have reached a level of sophistication which can not only reproduce many of the properties of today's galaxies, but also throws new light on high redshift galaxies which are too faint to be detected directly, such as those giving rise to quasar absorption lines. In this brief review, I summarise recent progress in these areas.
\end{abstract}

Keywords. galaxies: structure - galaxies: kinematics and dynamics - galaxies: evolution galaxies: high-redshift - (galaxies:) intergalactic medium

\section{Introduction}

In the last ten years we have witnessed a remarkable growth in the depth and breadth of galaxy redshift surveys which now stretch out to redshifts beyond $z=6$, sampling some $90 \%$ of the age of the universe. The underlying goal of such endeavours is to follow the process of galaxy formation and subsequent evolution, from the first objects to form at the end of the 'dark ages' all the way to the variety and beauty of the Hubble sequence of present-day galaxies. The kinematics of galaxies at different redshifts are of course a key ingredient of this whole story. In this brief review I shall concentrate on the epochs corresponding to $z=2-3$, where galaxy kinematics are increasingly drawing the attention of observers and theorists alike, now that a rough picture is taking shape of many of the physical properties of the varied galaxy populations already in place at that time.

The rotation curves of nearby spiral disks which we are all familiar with are assembled mostly from radio observations of atomic and molecular gas extending well beyond the luminous dimensions of galaxies. With the exception of sub-mm detected galaxies and AGN, current observational facilities lack the spatial resolution and sensitivity to conduct analogous measurements at high redshift. Instead, the internal kinematics of galaxies at $z=2-3$ are being probed via the narrow emission lines, primarily $\mathrm{H} \alpha$ and $[\mathrm{O}$ III $] \lambda 5007$, formed in the $\mathrm{H}$ II regions of galaxies during a period which saw the peak of the starformation activity in the cosmic history. At $z>1$, these nebular lines are redshifted into the near-infrared (near-IR), but are still detectable in the $J, H, K$ atmospheric windows, once the $\mathrm{OH}$ emission from the night sky has been carefully subtracted - an example is reproduced in Figure 1.

As we heard at this meeting, Bengt Strömgren was among the first to realise the value of mapping the distribution of ionised gas in the Milky Way via the Balmer lines. Half a century later, the technology available to today's astronomers makes it possible to extend the same quest to the distant universe, probing galaxies only 2-3 Gyr after the Big Bang. 

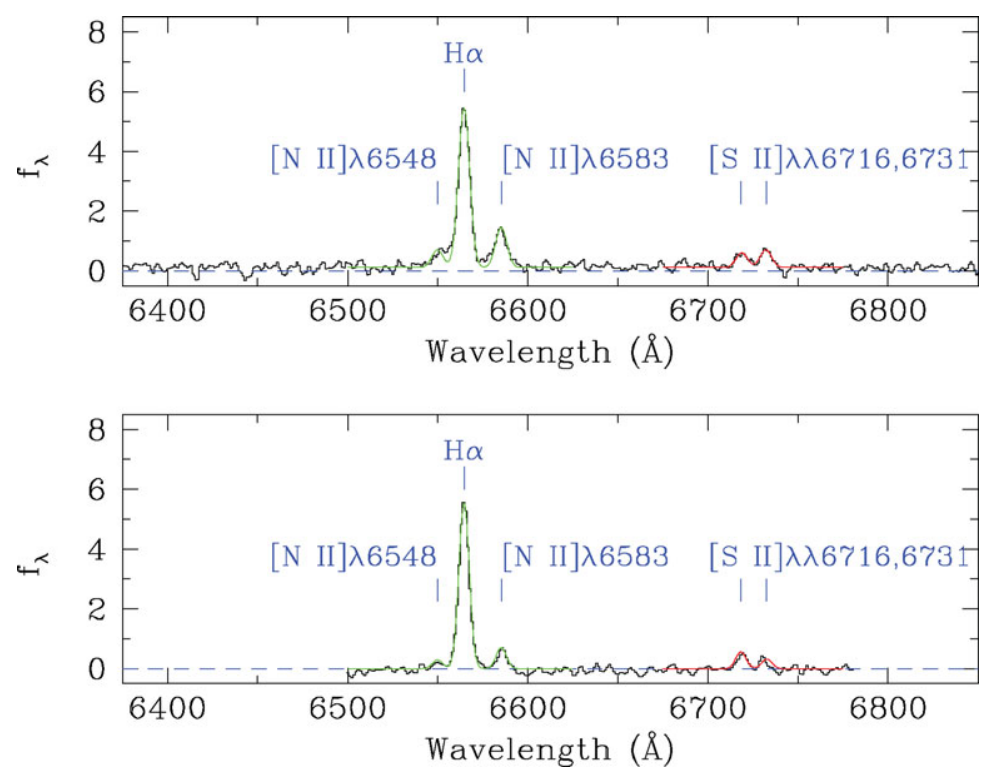

Figure 1. Composite spectra of star-forming galaxies at $z \simeq 2.2$ from the survey by Erb et al. (2006). At these redshifts, the emission lines (shown here at their rest-frame wavelengths) are redshifted into the near-IR $K$ band; these spectra were obtained with the NIRSPEC instrument on the Keck II telescope. Pettini \& Pagel (2004) proposed that the ratio of the [N II] and $\mathrm{H} \alpha$ emission lines, the $N 2$ index, is a convenient approximate measure of the nebular oxygen abundance. Galaxies brighter than $K_{s}=20$ (upper panel) have a mean ratio [N $\left.\mathrm{NI}\right] / \mathrm{H} \alpha=0.25$ which indicates an oxygen abundance $12+\log (\mathrm{O} / \mathrm{H})=8.56$, or $\sim 4 / 5$ solar, if the local calibration of the $N 2$ index with $(\mathrm{O} / \mathrm{H})$ applies to these galaxies. Galaxies fainter than $K_{s}=20$ (lower panel) have $[\mathrm{N}$ II $] / \mathrm{H} \alpha=0.13$ and $12+\log (\mathrm{O} / \mathrm{H})=8.39$ or $\sim 1 / 2$ solar.

\section{Near-Infrared Observations of Star-Forming Galaxies at $z=2-3$}

\subsection{Early Clues}

Hints of ordered motions within star-forming galaxies at $z=2-3$ were already present in the first near-IR observations on the ESO VLT with ISAAC, the first single-slit nearIR spectrograph to come on line on an 8-10 m telescope (Moorwood et al. 2000; Pettini et al. 2001). As the samples increased with the availability of NIRSPEC on the Keck II telescope, cases were commonly encountered where the redshifts of the $\mathrm{H} \alpha$ or [O III] lines varied continuously along the spectrograph slit, indicative of coherent shear which could be interpreted as rotation (Erb et al. 2003). However, it soon became apparent (Erb et al. 2004) that the velocity spread of the emission depended very sensitively on the seeing conditions under which the galaxies were observed. Furthermore, the lack of prior information on the morphologies of the galaxies targeted made it difficult to interpret such motions as rotation curves (despite some efforts by, for example, Weatherley \& Warren 2003), since the slit position angle on the sky was not necessarily aligned with the 'major axes' of the galaxies (see Figure 2).

\subsection{Integral Field Observations}

This area of study received a tremendous boost with the advent of SINFONI, the integral field near-IR spectrograph on the VLT. This instrument produced the first 'data-cubes' on galaxies at $z=2$, mapping their kinematics on the plane of the sky with an angular resolution of $\sim 0.5 \operatorname{arcsec}$, sampling spatial scales of a few kpc at these redshifts. An 

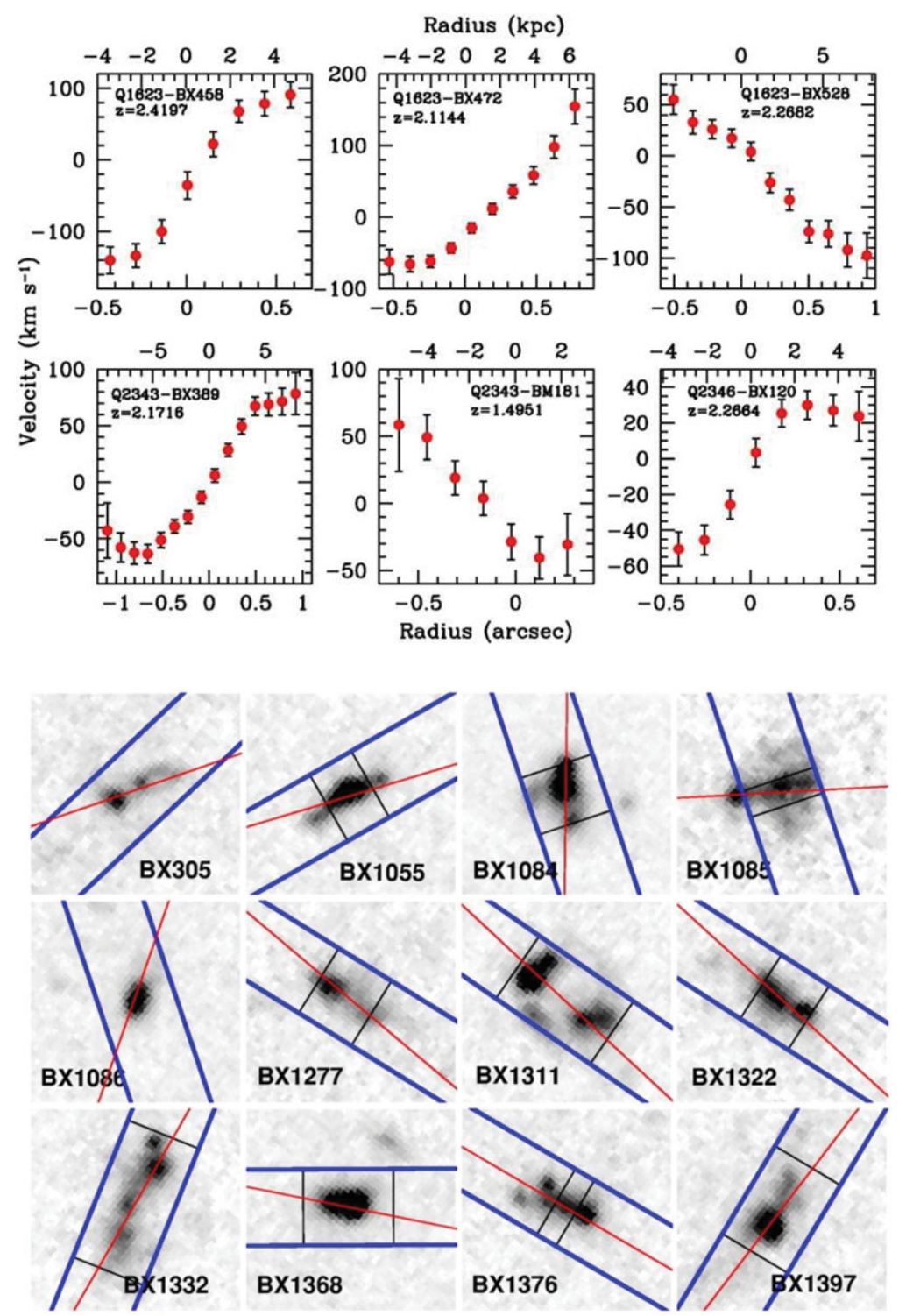

Figure 2. Upper Panel: 'Rotation curves' of $z \simeq 2$ galaxies derived from single-slit NIRSPEC observations of the $\mathrm{H} \alpha$ emission line. Lower Panel: HST ACS images of selected BX galaxies showing the size and orientation of the NIRSPEC entrance slit (blue lines). The slit is 0.76 arcsec wide, or $\sim 6 \mathrm{kpc}$ at the redshifts of the galaxies. (Both figures reproduced from Erb et al. 2004).

intensive observational programme described in R. Genzel's contribution to this volume, led to the first reports of galaxy kinematics which convincingly resemble rotation curves. In some of the brighter and more extended galaxies among the BX/BM sample of Steidel et al. (2004), the rotation curves could be followed to beyond $10 \mathrm{kpc}$ where they reached circular velocities as high as $v_{\mathrm{c}} \simeq 200 \mathrm{~km} \mathrm{~s}^{-1}$. In these well-developed systems, the implied dynamical masses seem to agree broadly with the stellar masses deduced from model-fitting of the rest-frame UV to near-IR spectral energy distributions, as expected 
Q1623-BX453 ( $z=2.1820)$
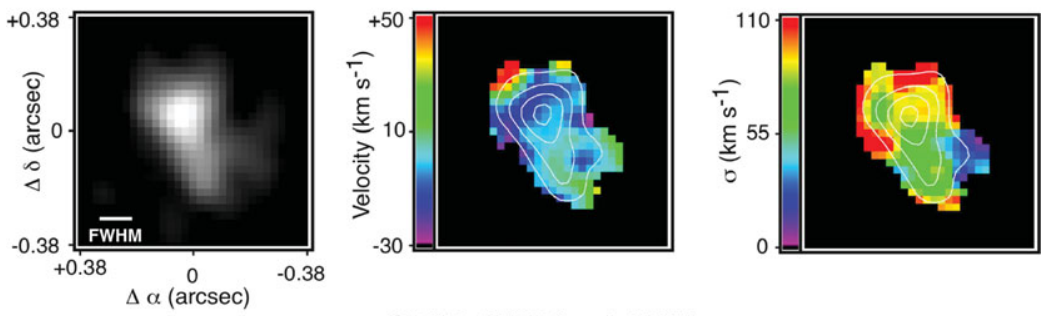

Q0449-BX93 ( $z=2.0067)$
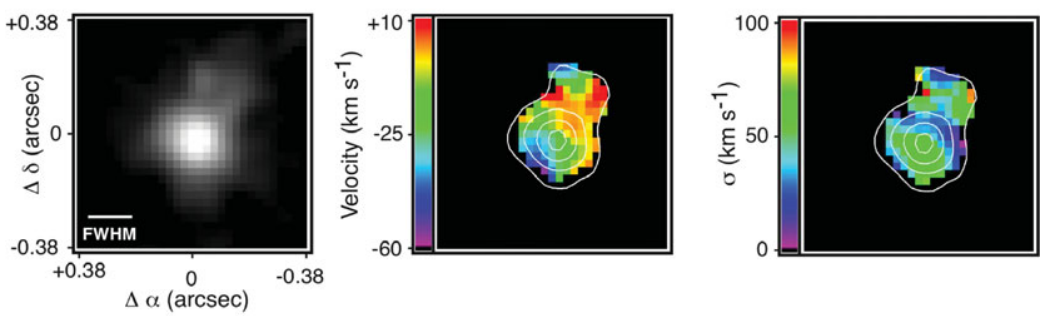

DSF2237a-C2 $(z=3.3172)$
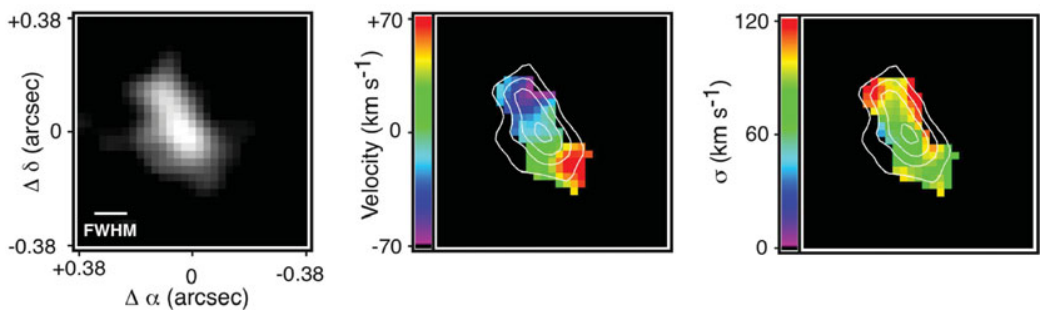

Figure 3. Maps obtained with the OH Suppressing InfraRed Imaging Spectrograph (OSIRIS) on the Keck II telescope (reproduced from Law et al. 2007a). For each of the three galaxies illustrated here, the maps show (from left to right): (a) the integrated flux in either the $\mathrm{H} \alpha$ or [O III] $\lambda 5007$ emission line; (b) the velocity of the emission line relative to systemic, according to the colour-coding shown on the left; and (c) the velocity dispersion. The field of view of $0.75 \times 0.75$ arcseconds of each panel corresponds to physical distances of $\sim 6 \mathrm{kpc}$ at the galaxies' locations. The resolution of the images is FWHM $=110-150$ mas, sampled with 25 mas pixels and shown by the white bar in the lower left-hand corner of the images in the first column.

if the baryons provide most of the gravitational potential in the inner regions of these galaxies.

The successful coupling of laser-guided adaptive optics to the near-IR integral field spectrograph OSIRIS on the Keck II telescope provided the next step forward by affording angular resolutions of $0.1-0.15$ arcsec, or $\sim 1 \mathrm{kpc}$ at $z=2-3$. Such fine spatial sampling pushes to the limit the light-gathering power of even $10 \mathrm{~m}$ telescopes; consequently, the galaxies initially targeted by Law et al. (2007a) were selected from the samples of Steidel et al. $(2003,2004)$ primarily on the basis of their high surface brightness (Law et al. 2007b).

Figure 3 shows examples of resolved kinematics in the inner regions $( \pm 3 \mathrm{kpc}$ from the centre) of three of the galaxies studied by Law et al. (2007b), illustrating the variety of patterns seen. In some cases the uniform progression from negative to positive velocities along the major axis is highly suggestive of rotation; the $z=3.3172$ galaxy DSF2237a-C2 shown in the bottom row of Figure 3 provides a good example of what could plausibly be interpreted as a rotating disk with $v_{\text {rot }} \simeq 70 \mathrm{~km} \mathrm{~s}^{-1}$. An analogous example at $z=1.5$ has 
been reported by Wright et al. (2007). If these are indeed disks, they are kinematically 'thick' in the sense that rotational and random velocities are of the same order: $v_{\text {rot }} / \sigma \approx 1$, as can be appreciated by comparing the middle and right panels of the bottom row in Figure 3 .

A counterexample is provided by the $z=2.1820$ galaxy Q1623-BX453 (top row of Figure 3). This UV-luminous galaxy is forming stars at a rate of $\sim 80 M_{\odot} \mathrm{yr}^{-1}$ and has already converted (by redshift $z=2.1820$ ) more than $50 \%$ of its available gas supply into stars, enriching its interstellar medium to near-solar metallicity (Erb et al. 2006). And yet, there is no indication of coherent kinematics in its inner regions, with hardly any velocity shear across the extent of the $\mathrm{H} \alpha$ emission (middle panel of the top row in Figure 3). We are either seeing this galaxy completely face-on, or here we have an example of a galaxy supported not by rotation but by random motions: the width of the resolved $\mathrm{H} \alpha$ emission line indicates velocity dispersions of $\sigma \simeq 60-100 \mathrm{~km} \mathrm{~s}^{-1}$ over most of the galaxy (see right-hand panel of the top row in Figure 3). Q1623-BX453 also provides an illustration of how, with the power of adaptive optics, we are beginning to discern inhomogeneities within the galaxies in the chemical composition of their H II regions (Figure 4). Similar examples of what could be the first indications of radial abundance gradients at high redshifts have been found by Förster Schreiber et al. (2006).

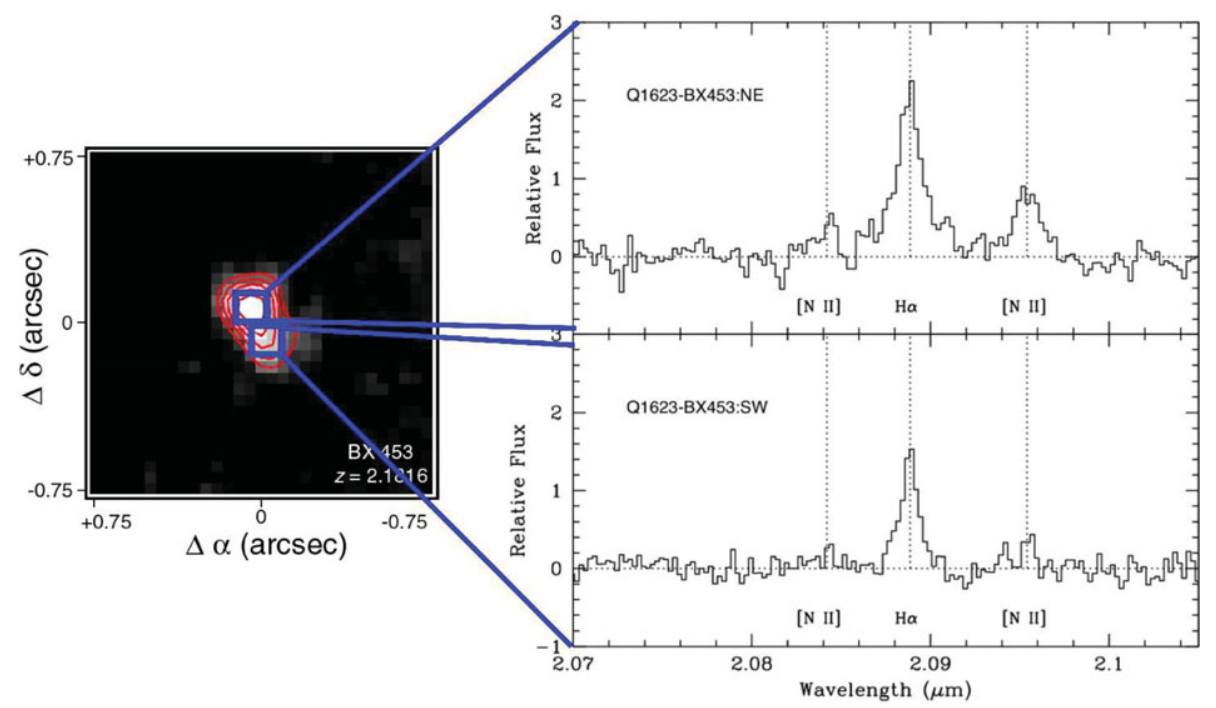

Figure 4. The brighter NE knot of $\mathrm{H} \alpha$ emission in the $z=2.1820$ galaxy Q1623-BX453 exhibits a high $[\mathrm{N}$ II $] / \mathrm{H} \alpha$ ratio indicative of a near-solar oxygen abundance. In the fainter knot, $<2 \mathrm{kpc}$ to the SW, Law et al. 2007a (from which this figure is reproduced) measured a lower value of the $N 2$ index, corresponding to $\mathrm{O} / \mathrm{H} \sim 1 / 2(\mathrm{O} / \mathrm{H})_{\odot}$.

In some cases, the improved resolution afforded by adaptive optics reveals morphologies and kinematics which are highly suggestive of on-going mergers. Figure 5 is a good example of a galaxy where the velocity shear first detected from single-slit spectra (Pettini et al. 2001) turned out to be due to two distinct 'clumps' (or in this case more likely two galaxies), each with a relatively high internal velocity dispersion $\left(\sigma \simeq 80 \mathrm{~km} \mathrm{~s}^{-1}\right)$ but separated by only a few tens of $\mathrm{km} \mathrm{s}^{-1}$ in redshift. 


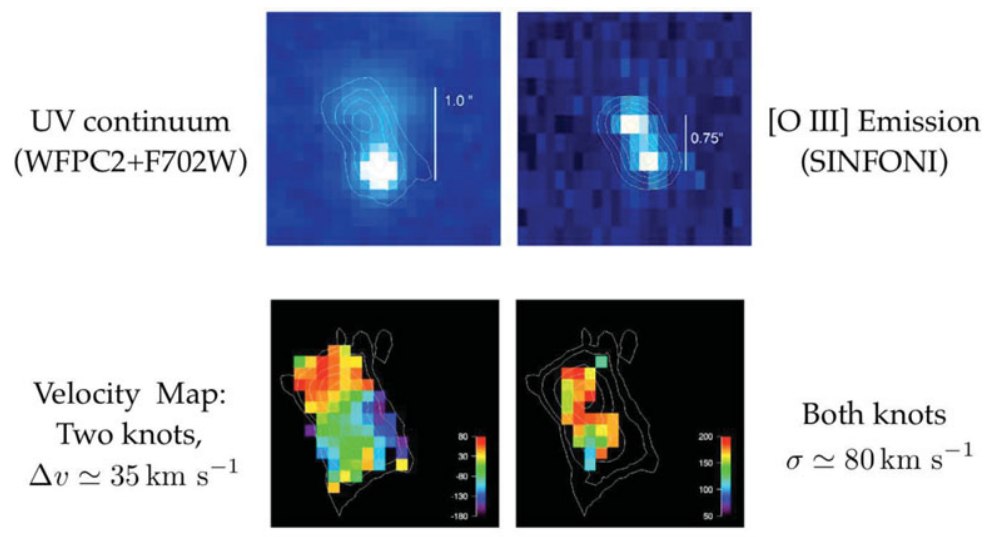

Q0347-383 C5, $z=3.2347$

Figure 5. Recent SINFONI observations of the $z=3.2347$ Lyman break galaxy Q0347-383 C5 by Nesvadba et al. 2008 (from which these figures are reproduced) show the source to consist of two merging Lyman break galaxies, separated by only $\sim 5 \mathrm{kpc}$. Other properties of these two galaxies seem to be typical of the LBG population, with star formation rates $\mathrm{SFR}=20-40 M_{\odot} \mathrm{yr}^{-1}$ and metallicities between solar and $1 / 5$ solar.

\subsection{Harnessing the Resolving Power of Gravitational Lenses}

In a few special instances it has proved possible to achieve spatial resolutions one order of magnitude higher than those of the data in Figures 3, 4 and 5, thereby sampling the internal structure of galaxies at $z=2-3$ on scales of only $\sim 100$ pc. Such remarkably sharp views are provided by the combination of adaptive optics and strong gravitational lensing by foreground clusters - with a good lens model it is possible to map the gravitationally
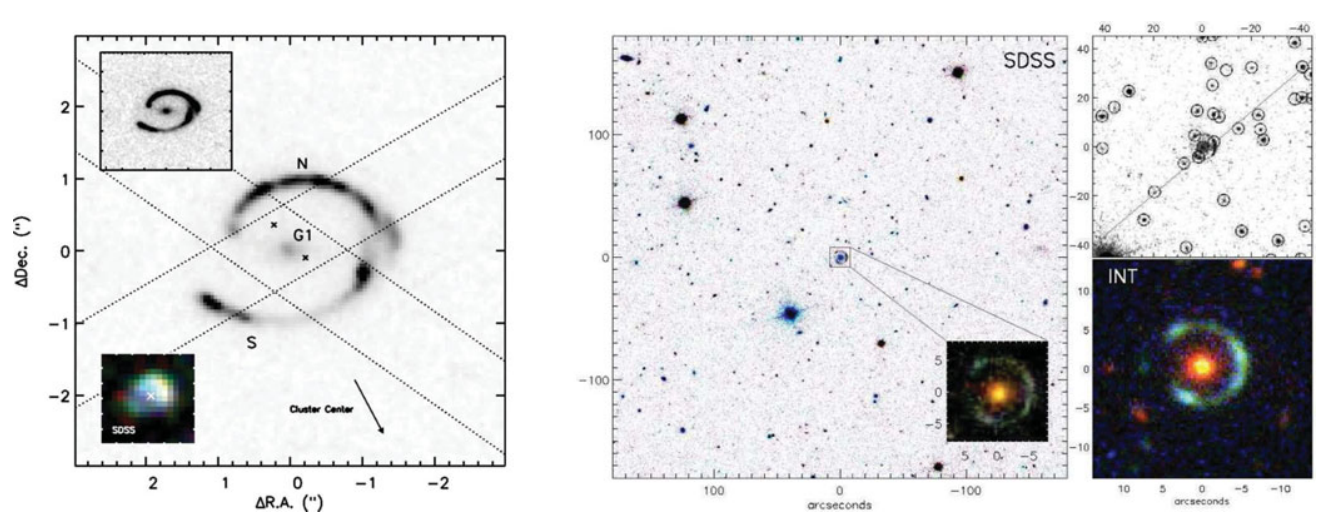

Figure 6. Two examples of recently discovered, strongly lensed, high redshift galaxies. Left Panel: the 'Cosmic Eye', a pair of high surface brightness arcs at $z_{\text {source }}=3.0735$ bracketing a foreground massive early-type galaxy at $z_{\text {lens }}=0.73$, discovered by Smail et al. (2007). Right Panel: the 'Cosmic Horseshoe', an almost complete ( 300 degrees) Einstein ring of diameter 10 arcsec discovered by Belokurov et al. (2007) from a targeted search in Sloan Digital Sky Survey Data Release 5 (SDSS DR5) images. The ring is due to a $z_{\text {source }}=2.379$ star-forming galaxy, magnified by a factor of $\sim 35$ by a $M \simeq 6 \times 10^{12} M_{\odot}$ foreground galaxy at $z_{\text {lens }}=0.444$. 
lensed images back onto the source plane at such extraordinary resolution. The number of strongly lensed high- $z$ galaxies has increased significantly in the last couple of years; there are now several examples known which appear as promising as the well studied MS 1512-cB58 (Pettini et al. 2002 and references therein).

Figure 6 shows two such examples where the fortuitous alignments of massive clusters (or a single massive galaxy) with background star-forming galaxies have produced nearcomplete Einstein rings. Stark et al. (in preparation) have used OSIRIS on the Keck II telescope to map the kinematics of the $z=3.0735$ galaxy lensed into the two 3 arcsec long arcs which make up the 'Cosmic Eye' (left panel of Figure 6 ). In the inner $\sim 2 \mathrm{kpc}$, the $\mathrm{H}$ II regions of the galaxy follow a rotation pattern which flattens at a projected distance from the centre of $r \sim 1 \mathrm{kpc}$ to $v_{\text {rot }} \sin i=54 \mathrm{~km} \mathrm{~s}^{-1}$. Again, random velocities are comparable to rotation $\left(v_{\text {rot }} / \sigma=0.9\right)$, so that it is by no means clear which mechanism is the dominant one in providing support for the baryons.

\section{Damped Lyman alpha Systems}

Long before we had learnt (or had the technological means) to recognise high redshift galaxies directly via their nebular and stellar emission, our knowledge of the 'normal' galaxy population at $z>1$ relied almost exclusively on the technique of QSO absorption line spectroscopy, illustrated in Figure 7. In particular, Art Wolfe and his collaborators

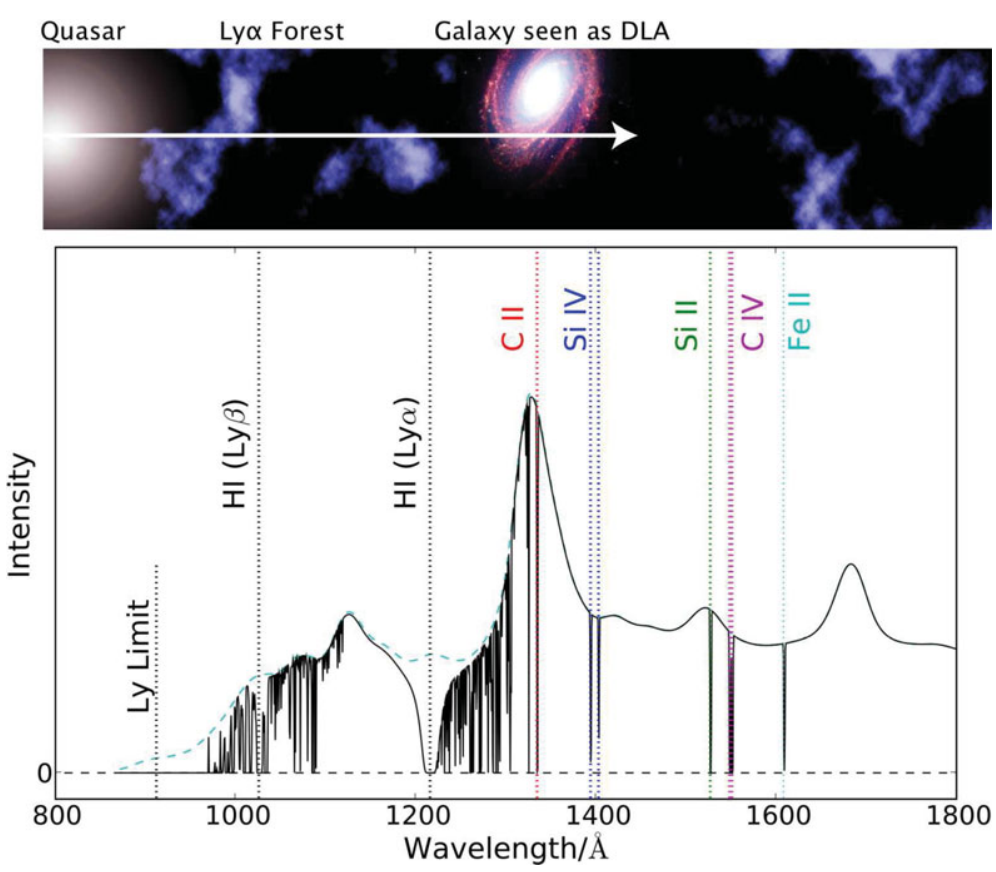

Figure 7. Illustration of the technique of QSO absorption line spectroscopy. The spectra of distant QSOs bear the imprint of intervening diffuse gas, cosmologically distributed along the line of sight, in the form of absorption lines. In the conventional view, low density gas in the intergalactic medium gives rise to the multitude of narrow absorption lines which constitute the Lyman alpha forest. The stronger, and less frequent, damped Lyman alpha lines, indicative of high column densities of neutral gas with $N(\mathrm{HI}) \geqslant 2 \times 10^{20}$ atoms $\mathrm{cm}^{-2}$, are thought to be the signposts of intervening galaxies intersected by the line of sight. Such absorption systems, or DLAs, include a variety of metal absorption lines due to the most common astrophysical elements. (Image courtesy of Andrew Pontzen). 


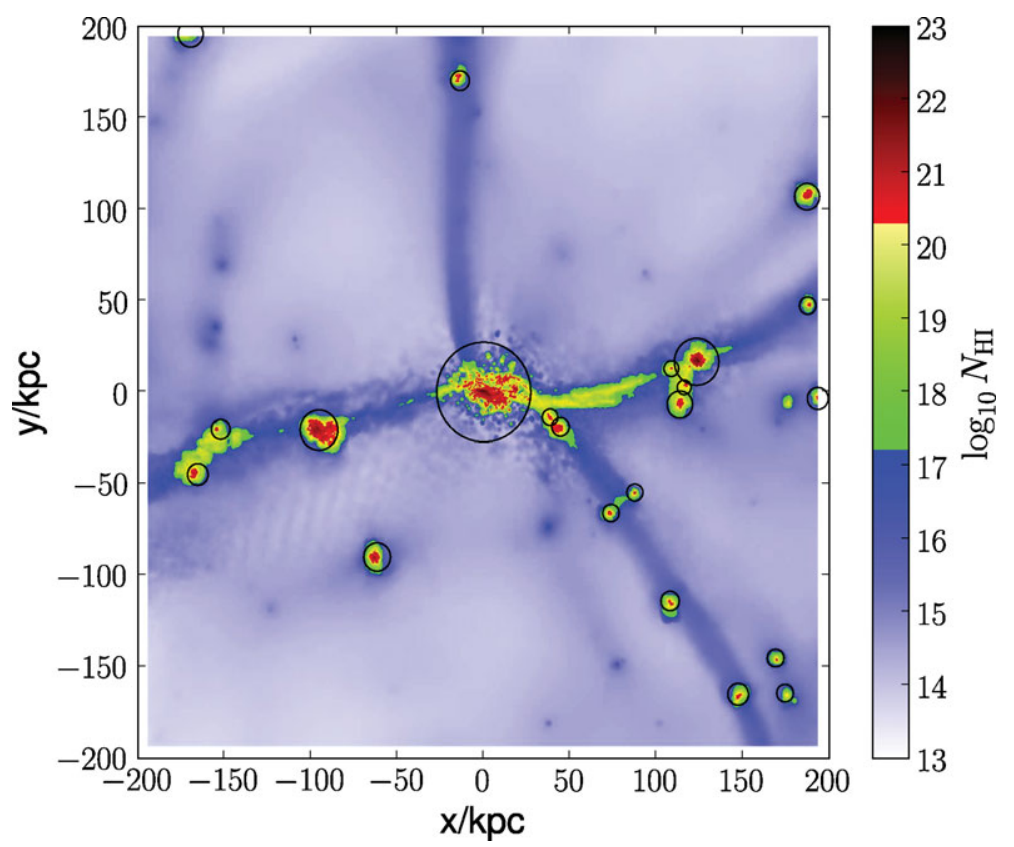

Figure 8. Two dimensional projection of the neutral hydrogen column density in a $400 \mathrm{kpc}$ cube centred on the major progenitor of a $z=0$ Milky Way-type galaxy in the simulations by Governato et al. (2007). The map is colour coded so that DLAs are dark red, while the lower column density Lyman limit systems (with $20.3>\log _{10}\left[N(\mathrm{H} \mathrm{I}) / \mathrm{cm}^{-2}\right]>17.2$ ) appear in green and yellow. The circles indicate the projected positions and virial radii of all dark matter halos with $M>5 \times 10^{8} M_{\odot}$. All units are physical. (Figure reproduced from Pontzen et al. 2008).

have long proposed the association of the strongest QSO absorbers, the damped Lyman alpha systems - or DLAs, with the progenitors of today's disk galaxies, observed at an early stage in the star formation process, when most of their baryons resided in their interstellar media (Prochaska \& Wolfe 1997, 1998). Empirical confirmation of this picture has, however, proved frustratingly difficult to attain. For one thing, the proximity of a bright QSO hinders the direct identification of the absorbers even in high resolution HST images and, for another, there is mounting evidence that DLAs sample a wide range of the galaxy luminosity function so that their numbers are dominated by galaxies fainter than $L^{*}$ (Wolfe, Gawiser, \& Prochaska 2005 and references therein).

At this meeting we have heard a great deal about simulations of galaxy formation in a cosmological context (reviewed in Simon White's contribution to these proceedings) which are increasingly sophisticated in resolution, volume, and treatment of relevant physical processes including the effects of star formation on the surrounding interstellar and intergalactic media (generally referred to as the 'feedback' of the star formation). It is only natural to ask what these 'model universes' tell us about DLAs and their association with galaxies. Pontzen et al. (2008) have recently revisited this question by analysing the output of the $N$-body simulations by the Seattle group (Governato et al. 2007 and references therein) which are among the most successful in reproducing many of the properties of disk galaxies at $z=0$ (e.g. Brooks et al. 2007; Governato et al. 2008).

Unlike previous simulations specifically aimed at DLAs (e.g. Razoumov et al. 2006; Nagamine et al. 2007 and references therein), there are no free parameters to be adjusted in the analysis by Pontzen et al. (2008) - these authors simply examined the properties of neutral gas in the volumes simulated by Governato et al. (2007) where relevant scaling 

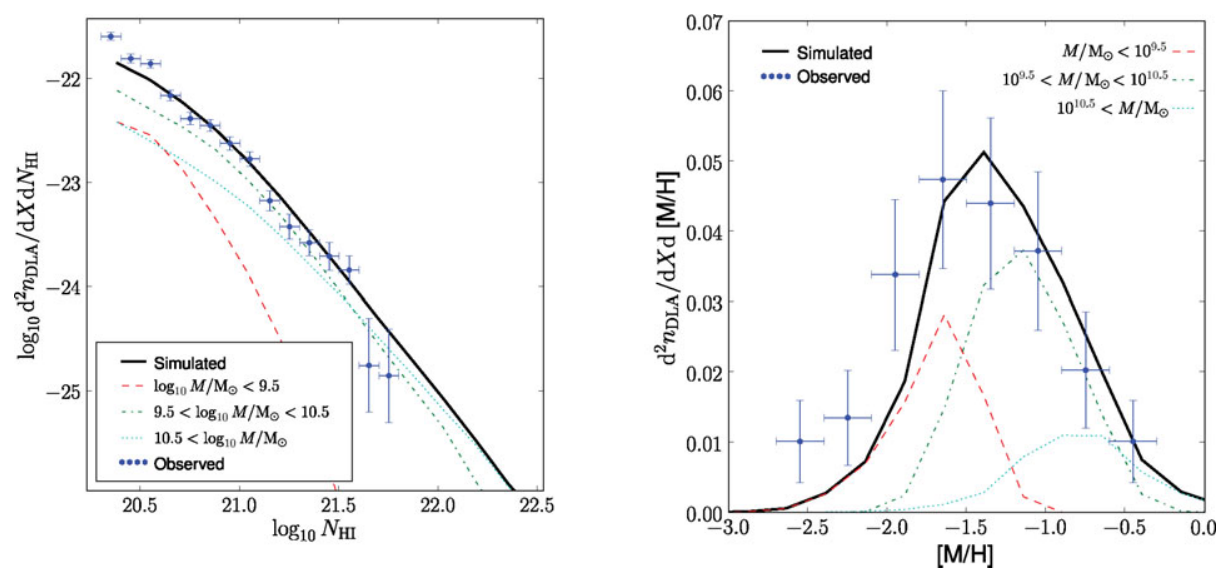

Figure 9. Comparisons between observed and simulated DLAs from the work by Pontzen et al. (2008) from which the figures are reproduced. Left Panel: the distribution of DLA column densities reported by Prochaska et al. (2005) from their analysis of SDSS DR5 quasar spectra is well matched by the incidence of DLAs in the volumes generated with the $N$-body simulations by Governato et al. (2007). Right Panel: The observed distribution of DLA metallicities (Prochaska et al. 2007) arises naturally in the Governato et al. (2007) disk galaxy simulations, without recourse to observationally unsubstantiated dust-biasing effects. In both figures the contributions from halos in different mass ranges are indicated separately, as well as their total.

parameters (most importantly the constant of proportionality in the Schmidt law of star formation and the efficiency of supernova energy deposition into the ISM) had already been fixed to the values that best reproduce the characteristics of disk galaxies today. The finding that the model galaxies generated with the code by Governato et al. (2007) do in fact account for many of the observed properties of DLAs without any fine tuning, as Figures 8, 9, and 10 illustrate, then lends support to the view that DLAs are an integral part of the galaxy formation process, and helps us towards the long-sought goal of making a connection between the properties of the absorbers and those of the galaxies producing them (Pettini 2004, 2006).

At $z=3$, random sightlines through the simulation volumes intersect neutral gas with the same frequency as the observed number of DLAs per unit redshift, implying effective radii of $\sim 10 \mathrm{kpc}$ (for column densities of neutral hydrogen $N(\mathrm{HI}) \geqslant 2 \times 10^{20} \mathrm{~cm}^{-2}$ ) in galaxies which by $z=0$ would grow to the mass of the Milky Way (see Figure 8 ). The most direct observational property of DLAs - their column density distribution, which has changed little since the original census by Wolfe et al. (1986), is reproduced very well (left panel of Figure 9), apart from the highest column density systems $(N(\mathrm{HI})>$ $5 \times 10^{21} \mathrm{~cm}^{-2}$ ) which are missing (compared to the model predictions) from even the largest quasar surveys, such as the SDSS (Prochaska et al. 2005). Possibly, the gas is mostly molecular and very short-lived (i.e. it turns into stars) when such high surface densities are reached (Schaye 2001).

For the first time, the observed distribution of DLA metallicities is reproduced reasonably well (right panel of Figure 9). Given the substantial uncertainties involved in, for example, the adopted stellar yields of Type II and Type Ia supernovae, the initial stellar mass function, and even the solar abundance scale used for comparison, the agreement may be somewhat fortuitous. However, it is significant that the good match in Figure 9 

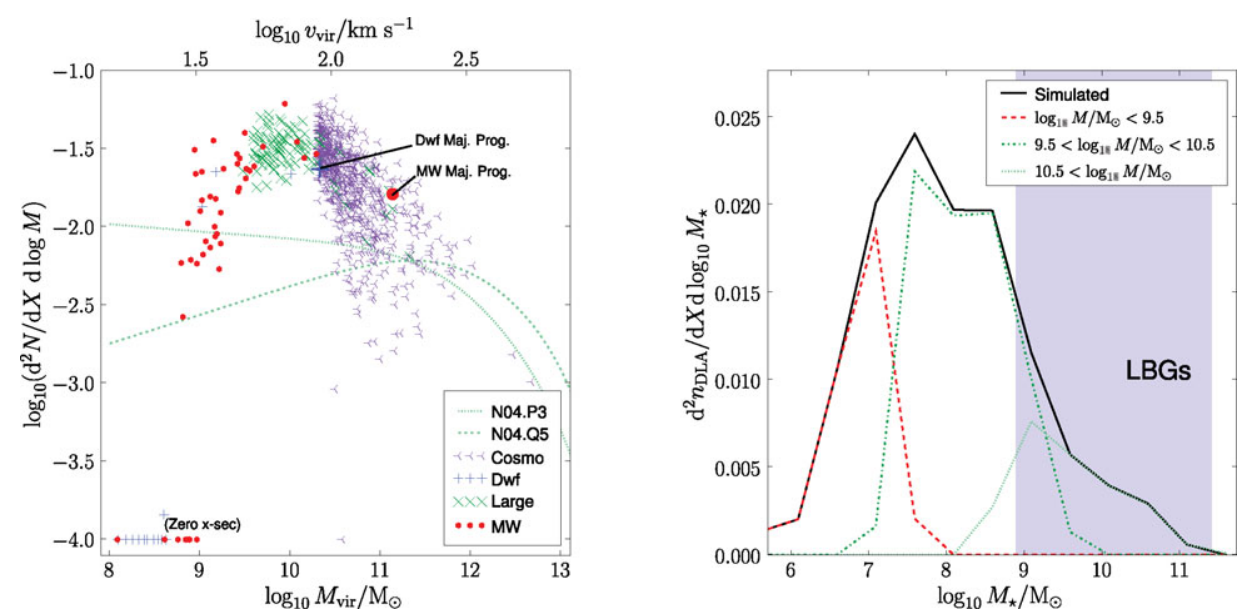

Figure 10. Contributions by different galaxies to the total DLA cross-section on the sky at $z=3$ in the work by Pontzen et al. (2008) from which the figures are reproduced. Left Panel: Relative contributions to the DLA cross-section by halos of different masses (lower $x$-axis) or virial velocities (top axis). Results from different simulation volumes are colour-coded (see Pontzen et al. 2008 for further details). Halos which do not contain significant column densities of neutral hydrogen, and thus do not give rise to DLAs, are shown artificially at -4 on the $y$-axis. Also shown for comparison are the relative contributions from galaxies in the earlier simulations by Nagamine et al. (2004). Right Panel: Fractional contributions from galaxies of different stellar masses assembled by $z=3$. Most DLAs arise in galaxies which are less massive and fainter than the Lyman break galaxies surveyed by Steidel and collaborators (Steidel et al. 2003; Shapley et al. 2001).

is achieved without appealing to a 'hidden' population of metal- and dust-rich DLAs which could potentially exclude background QSOs from current, optically-selected, samples. Many previous DLA simulations, which tended to overestimate the frequency of high-metallicity DLAs, have invoked such a scenario to reconcile their predictions with the data, overlooking at times the fact that this supposed source of bias has been shown to be of minor importance by DLA surveys in radio selected QSOs (Ellison et al. 2001, 2005; Jorgenson et al. 2006).

As discussed by Pontzen et al. (2008), the good agreement between theoretical and observed metallicity distributions is achieved naturally in the Governato et al. (2007) simulations because a substantial fraction of the DLA cross-section is produced by halos of relatively low mass, with virial masses $10^{9.5} M_{\odot} \leqslant M_{\mathrm{vir}} \leqslant 10^{10.5} M_{\odot}$ (see left panel of Figure 10), which have undergone relatively little star formation by $z=3$ and whose shallow potential wells probably retain only a small fraction of the heavy elements produced (see Julianne Dalcanton's contribution). Such galaxies make up the dominant contribution to the DLA cross-section partly because their interstellar gas is 'puffed up' to larger radii than it would otherwise be the case by the inclusion of feedback in the galaxy formation prescription.

If the simulations by Governato et al. (2007) offer a realistic representation of the connection between DLAs and galaxies, as the analysis by Pontzen et al. (2008) seems to indicate, we can tentatively associate subsets of DLAs chosen according to a particular characteristic with their host halos. For example, Pontzen et al. (2008) find a broad mass-metallicity relation in their simulations, with the least metal-enriched DLAs tracing preferentially low-mass halos (see right panel of Figure 9). Figure 8 gives a visualisation 
of such halos clustered around the progenitor of a Milky Way-type galaxy. These systems may be some of the best sites where to look for chemical traces of the 'first stars', as well as the unadulterated products of primordial nucleosynthesis (Pettini et al. 2008a,b).

\section{Conclusions}

Continuing advances in observational and computational facilities are giving us clearer views of galaxies at high redshifts than ever before. The recently gained ability to probe the internal kinematics of galaxies at look-back times of more than $75 \%$ of the age of the universe is as powerful a demonstration as any that laser-guided adaptive optics has finally come of age and can be successfully used to feed high resolution spectrographs. The picture that is emerging is a complex one. The finding that major mergers were common at $z=2-3$ comes as no surprise. However, there are also many examples of galaxies already exhibiting large-scale rotation, which somewhat remarkably appears not to have been disrupted over a period of several hundred million years, during which the galaxies have accreted a large fraction of their baryonic mass, assembling as much as $M_{*} \sim 5 \times 10^{10} M_{\odot}$ in stars (Genzel et al. 2006). Clarifying the relative incidence of these different kinematic signatures remains a priority for future observations, given their potential for discriminating between different scenarios of gas accretion and cooling within dark matter halos (e.g. Immeli et al. 2004; Dekel \& Birnboim 2006).

On the theoretical front, we have yet to develop a unified picture of the different kinematic components of high- $z$ galaxies, which presumably include: (a) infalling gas fuelling star formation, (b) rotation, (c) large velocity dispersions, and (d) galaxy-wide outflows of the interstellar medium driven by the energy and momentum released by the star formation activity. In particular, what is the origin of the high turbulence which seems to be present in almost every case, irrespectively of whether a rotation pattern can be discerned or not? Which is the dominant support mechanism in these galaxies? And why is it so difficult to identify the infalling gas, when the observational signatures of the outflows are so obvious? The work by Pontzen et al. (2008) strongly suggests that our Milky Way had the characteristics of a damped Lyman alpha system at $z=3$, but what fraction of the galaxies we see directly at these redshifts evolve to become today's large spirals?

What I took away from this meeting is the high level of interest, both observational and theoretical, in exploring these questions. The goal of bringing together the different pieces of the jig-saw puzzle of galaxy formation is within sight and we can look forward to a time in the not too distant future when the legacy of the work begun by Bengt Strömgren will be fulfilled.

\section{Acknowledgements}

It is a pleasure to acknowledge all of my collaborators in the various projects described here; I especially thank David Law and Andrew Pontzen who very generously provided me with much of the material for my presentation. I, like the rest of the participants of this stimulating meeting in the beautiful city of Copenhagen, am indebted to Johannes Andersen and Birgitta Nordström whose unflagging efforts and excellent organisation made the symposium such a success.

\section{References}

Belokurov, V., et al. 2007, ApJ, 671, L9

Brooks, A. M., et al. 2007, ApJ, 655, L17 
Dekel, A. \& Birnboim, Y. 2006, MNRAS, 368, 2

Ellison, S. L., Yan, L., Hook, I. M., Pettini, M., Wall, J. V., \& Shaver, P. 2001, A\&\&A, 379, 393 Ellison, S. L., Hall, P. B., \& Lira, P. 2005, AJ, 130, 1345

Erb, D. K., Shapley, A. E., Steidel, C. C., Pettini, M., Adelberger, K. L., Hunt, M. P., Moorwood, A. F. M., \& Cuby, J.-G. 2003, ApJ, 591, 101

Erb, D. K., Steidel, C. C., Shapley, A. E., Pettini, M., \& Adelberger, K. L. 2004, ApJ, 612, 122

Erb, D. K., Steidel, C. C., Shapley, A. E., Pettini, M., Reddy, N. A., \& Adelberger, K. L. 2006, ApJ, 646, 107

Förster Schreiber, N. M. et al. 2006, ApJ, 645, 1062

Genzel, R. et al. 2006, Nature, 442, 786

Governato, F., Mayer, L., \& Brook, C. 2008, ArXiv e-prints, 801, arXiv:0801.1707

Governato, F., Willman, B., Mayer, L., Brooks, A., Stinson, G., Valenzuela, O., Wadsley, J., \& Quinn, T. 2007, MNRAS, 374, 1479

Immeli, A., Samland, M., Gerhard, O., \& Westera, P. 2004, A\&SA, 413, 547

Jorgenson, R. A., et al. 2006, ApJ, 646, 730

Law, D., Steidel, C. C., Erb, D. K, Larkin, J. E., Pettini, M., Shapley, A. E., \& Wright, S. A. 2007a, ApJ, 669, 929

Law, D. R., Steidel, C. C., Erb, D. K., Pettini, M., Reddy, N. A., Shapley, A. E., Adelberger, K. L., \& Simenc, D. J. 2007b, ApJ, 656, 1

Moorwood, A. F. M., van der Werf, P. P., Cuby, J. G., \& Oliva, E. 2000, A\&\&A, 362, 9

Nagamine, K., Springel, V., \& Hernquist, L. 2004, MNRAS, 348, 421

Nagamine, K., Wolfe, A. M., Hernquist, L., \& Springel, V. 2007, ApJ, 660, 945

Nesvadba, N. P. H., Lehnert, M., Davies, R., Verma, A., \& Eisenhauer, F. 2008, A\&BA, 479, 67

Pettini, M. 2004, in C. Esteban, R. J. García López, A. Herrero \& F. Sánchez (eds.), Cosmochemistry. The melting pot of the elements (Cambridge: Cambridge University Press), p. 257 (astro-ph/0303272)

Pettini, M. 2006 in V. LeBrun, A. Mazure, S. Arnouts \& D. Burgarella (eds.), The Fabulous Destiny of Galaxies: Bridging Past and Present (Paris: Frontier Group), p. 319 (astro$\mathrm{ph} / 0603066)$

Pettini, M. \& Pagel, B. E. J. 2004, MNRAS, 348, L59

Pettini, M., Rix, S. A., Steidel, C. C., Adelberger, K. L., Hunt, M. P., \& Shapley, A. E. 2002, ApJ, 569, 742

Pettini, M., Shapley, A. E., Steidel, C. C., Cuby, J.-G., Dickinson, M., Moorwood, A. F. M., Adelberger, K. L., \& Giavalisco, M. 2001, ApJ, 554, 981

Pettini, M., Zych, B. J., Murphy, M. T., Lewis, A., \& Steidel, C. C. 2008a, MNRAS, in press

Pettini, M., Zych, B. J., Steidel, C. C., \& Chaffee, F. H. 2008b, MNRAS, 385, 2011

Pontzen, A., Governato, F., Pettini, M., et al. 2008, MNRAS, in press

Prochaska, J. X., Herbert-Fort, S., \& Wolfe, A. M. 2005, ApJ, 635, 123

Prochaska, J. X. \& Wolfe, A. M. 1997, ApJ, 487, 73

Prochaska, J. X. \& Wolfe, A. M. 1998, ApJ, 507, 113

Prochaska, J. X., et al. 2007, ApJ Supp, 171, 29

Razoumov, A. O., Norman, M. L., Prochaska, J. X., \& Wolfe, A. M. 2006, ApJ, 645, 55

Schaye, J. 2001, ApJ, 562, L95

Shapiro, K. L., et al. 2008, ApJ, in press

Shapley, A. E., Steidel, C. C., Adelberger, K. L., Dickinson, M., Giavalisco, M., \& Pettini, M. 2001, ApJ, 562, 95

Smail, I., et al. 2007, ApJ, 654, L33

Steidel, C. C., Adelberger, K. L., Shapley, A. E., Pettini, M., Dickinson, M., \& Giavalisco, M. 2003, ApJ, 592, 728

Steidel, C. C., Shapley, A. E., Pettini, M., Adelberger, K. L., Erb, D. K., Reddy, N. A. \& Hunt, M. P. 2004, ApJ, 604, 534

Weatherley, S. J. \& Warren, S. J. 2003, MNRAS, 345, L29

Wolfe, A. M., Gawiser, E., \& Prochaska, J. X. 2005, ARAA, 43, 861

Wolfe, A. M., Turnshek, D. A., Smith, H. E., \& Cohen, R. D. 1986, ApJ Supp, 61, 249

Wright, S. A., et al. 2007, ApJ, 658, 78 